\title{
Article \\ Photocatalytic polymeric nanocomposite membrane towards oily wastewater
}

\author{
D. Zioui ${ }^{1 \dagger}$, H. Salazar ${ }^{2,3+}$, L. Aoudjit1 ${ }^{1,4}$, P. M. Martins' ${ }^{2,3^{*}}$, and S. Lanceros-Méndez ${ }^{2,5,6^{*}}$ \\ ${ }^{1}$ Unité de Développement des équipements Solaires, UDES /Centre de Développement des Energies \\ Renouvelables, CDER, Bou Ismail, 42415, W. Tipaza, Algérie \\ ${ }^{2}$ Centre/Departament of Physics, University of Minho, Campus de Gualtar, 4710-057 Braga, Portugal \\ ${ }^{3}$ Institute of Science and Innovation on Bio-Sustainability (IB-S), University of \\ Minho, 4710-057, Braga, Portugal \\ ${ }^{4}$ Laboratoire de Chimie du Gaz Naturel, Faculté de Chimie, BP 32, 16111, El Alia, U.S.T.H.B., Bab Ezzouar, \\ Algérie \\ ${ }^{5}$ BCMaterials, Basque Centre for Materials, Applications and Nanostructures, UPV/EHU Science Park, 48940 \\ Leioa, Spain
}

6IKERBASQUE, Basque Foundation for Science, 48013 Bilbao, Spain

\footnotetext{
* Author to whom correspondence should be addressed; E-Mail: senentxu.lanceros@bcmaterials.net and pamartins@fisica.uminho.pt
}

\begin{abstract}
Production of wastewater related to the oil and gas industries is increasing over the years. The compounds found in industrial wastewater typically show high toxicity, and in this way, they have become a primary environmental concern. Several techniques have been applied in industrial effluents remediation. In spite of the efforts, these techniques are yet ineffective to treat oily wastewater before it can be discharged safely to the environment. Membrane technology is an attractive approach to treat oily wastewater. This is dedicated to the immobilisation of $\mathrm{TiO}_{2}$ nanoparticles on poly (vinylidene fluoride-trifluoro ethylene) (PVDF-TrFE) porous matrix by solvent casting. Membranes with interconnected pores with an average diameter of 60 micrometres and the contact angle of $97^{\circ}$, decorated with $\mathrm{TiO} 2$ nanoparticles, are obtained. The degradation of oily wastewater demonstrated the remarkable photocatalytic efficiency of the nanocomposite membranes: under sunlight irradiation for 7 hours, colourless water was obtained. These results show the suitability of $\mathrm{TiO}_{2} / \mathrm{P}(\mathrm{VDF}-\mathrm{TrFE})$ nanocomposite for photocatalytic applications for oily wastewater remediation.
\end{abstract}

Keywords: oil, nanocomposite membranes, PVDF-TrFE, photoreactor, and sunlight.

\section{Introduction}

Oil contaminated water is one of the most concerning pollution sources worldwide. The production of oil and gas by petroleum refineries yields to a large amount of wastewater, containing essentially by organic compounds (aliphatic and aromatic hydrocarbons), inorganic compounds (metals), dissolved and suspended solids(Rusli, Alias, Shahruddin and Othman, 2016).

Nowadays, many techniques are being employed in oily wastewater treatment, such as gravity separation (Sun, Xiao, Zhou, Wei, Shi and Jiang, 2018), ultrasonic separation (Stack, Carney, Malone and Wessels, 2005), adsorption (Soares, Rodrigues, Trindade and Daniel-da-Silva, 2017), coagulation/flocculation (Cañizares, Martínez, Jiménez, Sáez and Rodrigo, 2008), among others. However, these techniques present some drawbacks, like the large space requirement, high low cost 
and the generation of secondary pollutants. On the other hand, membrane processes are considered a suitable alternative for oily wastewater treatment (Ong, Lau, Goh, Ng and Ismail, 2014). Membranes based on poly(vinylidene fluoride), PVDF, and its copolymers are of high interest for wastewater treatment, due to its outstanding properties, like mechanical and chemical resistance and hydrolytic and thermal stability (Salazar, Nunes-Pereira, Correia, Cardoso, Gonçalves, Martins, Ferdov, Martins, Botelho and Lanceros-Méndez, 2016). Membranes based on PVDF have been investigated for the removal of different pollutants from water, such as copper ions (Zhang, Wang, Liu, Xu, Han and $\mathrm{Xu}, 2014$ ), natural organic matter (Song, Shao, Wang and Zhong, 2014), proteins (Zhang, Zhang, Cheng, Xu, Xu, Chen, Lai and Tung, 2012), volatile organic compounds (Ramaiah, Satyasri, Sridhar and Krishnaiah, 2013) and desalination (Zuo, Shi, Tian, Yu, Wang and He, 2013), among others. From all the PVDF copolymers, poly (vinylidene fluoride-trifluoroethylene) presents suitable physicochemical properties for photocatalytic applications, like a high UV resistance. Furthermore, it allows the production of membranes with controlled porosity and pore size, possesses good chemical, thermal and mechanical resistance (Aoudjit, Martins, Madjene, Petrovykh and LancerosMendez, 2018). Also, this copolymer has shown remarkable photocatalytic activity in degradation of organic compounds such as methylene blue (Martins, Gomez, Lopes, Tavares, Botelho, Irusta and Lanceros-Mendez, 2014) and tartrazine (Aoudjit, Martins, Madjene, Petrovykh and LancerosMendez, 2018).

Titanium dioxide $\left(\mathrm{TiO}_{2}\right)$ is the most widely used photocatalyst for degradation of organic compounds due to its properties, such as non-toxicity, low cost and abundance, physical and chemical stability, superhydrophilicity, and superior photocatalytic activity under UV light irradiation ( $\lambda \mathrm{max}=390 \mathrm{~nm})$ (Martins, Miranda, Marques, Tavares, Botelho and Lanceros-Mendez, 2016). The incorporation of $\mathrm{TiO}_{2}$ into polymeric membranes solve some drawbacks related to its use in suspension, namely the recuperation of the particles. Previously, $\mathrm{TiO}_{2} / \mathrm{PVDF}-\mathrm{TrFE}$ nanocomposite membranes proved to be suitable for wastewater treatment, due to its physicochemical properties (excellent mechanical, thermal, chemical and UV radiation resistance), and have been proven to show remarkable photocatalytic activity in the degradation of tartrazine, with sunlight photocatalytic activity of $78 \%$, over 5 hours (Aoudjit, Martins, Madjene, Petrovykh and Lanceros-Mendez, 2018).

Photocatalytic membrane reactors (PMR) are an efficient, environmentally friendly and low-cost wastewater treatment technique. PMR have shown great potential as a "zero" waste process for oily wastewater treatment, since they can reduce the loss of photocatalyst, to control the contact time between catalyst and pollutant, and to realise a continuous process. Because of that, PMR can improve the process efficiency and stability, and reuse the catalyst to reduce operating cost (Zhang, Ding, Luo, Jaffrin and Tang, 2016).

Thus, the goal of this work is to demonstrate the suitability of $\mathrm{TiO}_{2}$ nanoparticles immobilised on a $\mathrm{P}(\mathrm{VDF}-\mathrm{TrFE})$ porous membrane as an efficient method for the degradation of oil in wastewater in a solar photoreactor.

\section{Results and Discussion}

The nanoparticles and membrane characterisation are summarised in Figure 1. The crystalline structure of $\mathrm{TiO}_{2}$ nanoparticles was assessed by X-ray diffraction (XRD) and compared with the pattern diffractograms (Figure 1a). The XRD pattern shows intense diffraction peaks at $25^{\circ}$ and $48^{\circ}$ 
indicating $\mathrm{TiO}_{2}$ in the anatase phase. Additionally, diffractogram also exhibits intense diffraction peaks at $27^{\circ}, 36^{\circ}$, and $55^{\circ}$ that indicates $\mathrm{TiO}_{2}$ in the rutile phase. The peaks are similar to the standard spectra (JCPDS nos:88-1175 and 84-1286) (Jin, Duan, Liu, Chen, Yang and Guo, 2015).

SEM cross-section images, Figure $1 \mathrm{~b}$, shows the porous microstructure of the produced membrane with the characteristic interconnected (small pores inside the pores) spherical pores with an average diameter of $\approx 60 \mu \mathrm{m}$. Moreover, it is observed the presence of $\mathrm{TiO}_{2}$ agglomerates inside the pores and attached to the pore walls (inset of Figure $1 b$ ).

Infrared spectroscopy (FTIR) was used to determine the polymer phase and the possible chemical interaction between fillers and polymer matrix. In Figure 1c, the FTIR spectra of 8 wt. \% TiO $/ \mathrm{P}(\mathrm{VDF}-$ $\mathrm{TrFE}$ ) nanocomposite show a stable polymer structure concerning the pure polymer. Bands at 840 , 1288 and $1400 \mathrm{~cm}^{-1}$ show that the polymer crystallises in the all-trans piezoelectric $\beta$-phase (Ribeiro, Costa, Correia, Nunes-Pereira, Oliveira, Martins, Gonçalves, Cardoso and Lanceros-Méndez, 2018).
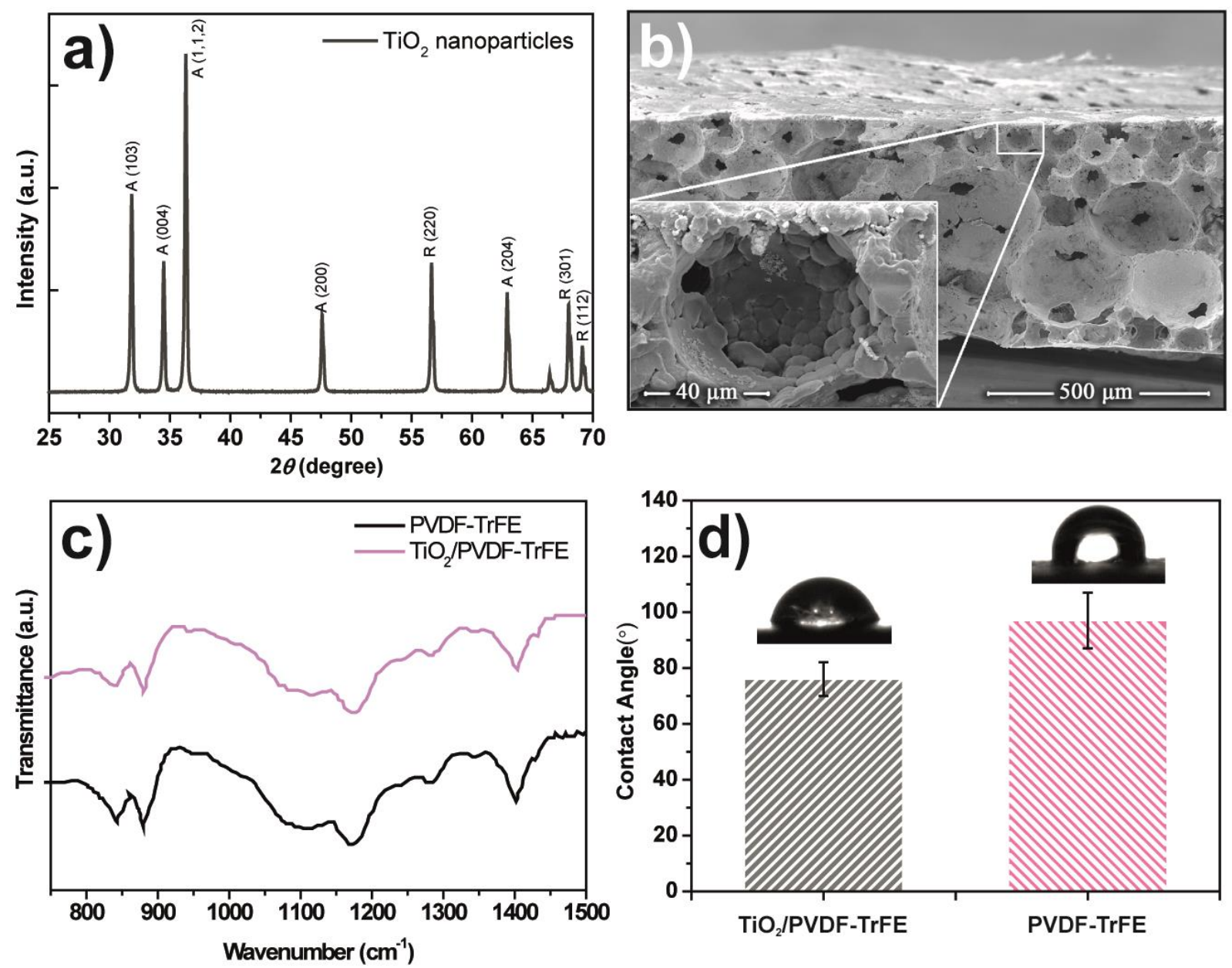

Figure 1 - XRD pattern of $\mathrm{TiO}_{2}$ nanoparticles and identification of different crystal phases: anatase (A), and rutile (R) a). Cross-section SEM images of 8 wt. \% $\mathrm{TiO}_{2} / \mathrm{P}(\mathrm{VDF}-\mathrm{TrFE})$ membranes with an inset showing a detail of an interconnected pore b). FTIR spectra and contact angle of $8 \mathrm{wt}$ \% $\mathrm{TiO}_{2} / \mathrm{P}(\mathrm{VDF}-\mathrm{TrFE})$ nanocomposite membranes and pure polymer, c) and d), respectively.

The polymer phase remains unchanged when $\mathrm{TiO}_{2}$ is present in the membranes, as already reported in Martins et al. (Martins, Miranda, Marques, Tavares, Botelho and Lanceros-Mendez, 2016). In this 
way, filler content and type do not change the crystallisation phase of the polymer, which crystallises in the all-trans $\beta$-phase and no chemical bonds are detected between polymer and fillers.

Contact angle measurements assessed the wettability of the membranes. Figure $1 \mathrm{~d}$ illustrates the contact angle for the pristine and the $8 \%$ of $\mathrm{TiO}_{2} / \mathrm{PVDF}-\mathrm{TrFE}$ samples, which presents contact angles of 97 and aa $76^{\circ}$, respectively. These results show the decrease of the contact angle with the incorporation of $\mathrm{TiO}_{2}$ nanoparticles on the polymer matrix, which in turn, allows a for a higher interaction between the nanocatalyst and the oily water.

The polymer phase remains unchanged when $\mathrm{TiO}_{2}$ is present in the membranes, as already reported in Martins et al. (Martins, Miranda, Marques, Tavares, Botelho and Lanceros-Mendez, 2016). In this way, filler content and type do not change the crystallisation phase of the polymer, which crystallises in the all-trans ๑-phase and no chemical bonds are detected between polymer and fillers.

Contact angle measurements assessed the wettability of the membranes. Figure $2 \mathrm{~d}$ illustrates the contact angle for the pristine and the $8 \%$ of $\mathrm{TiO}_{2} / \mathrm{PVDF}-\mathrm{TrFE}$ samples, which presents contact angles of 97 and aa $76^{\circ}$, respectively. These results show the decrease of the contact angle with the incorporation of $\mathrm{TiO}_{2}$ nanoparticles on the polymer matrix, which in turn, allows a for a higher interaction between the nanocatalyst and the oily water.

\subsection{Photocatalytic degradation of oily wastewater}

The $8 \% \mathrm{TiO}_{2} / \mathrm{P}(\mathrm{VDF}-\mathrm{TrFE})$ membranes were used to assess the degradation of oily wastewater in a solar photoreactor. Figure 2 shows the shift of transparency of the oily water after 7 hours under sunlight radiation. The visual inspection indicates that the oily wastewater pollutants were degraded as transparent water was obtained after the 7 hours.

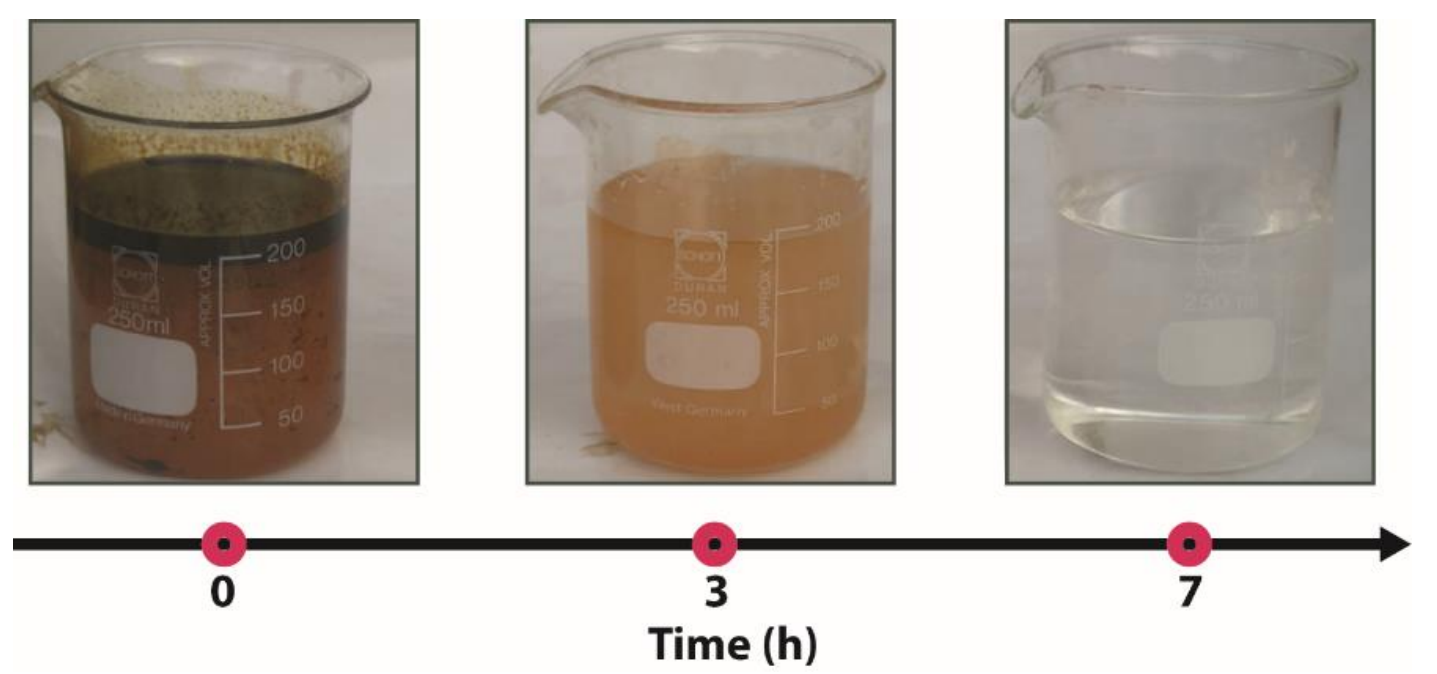

Figure 2 - Appearance of the oily wastewater along the treatment with the 8 wt. \% $\mathrm{TiO}_{2} / \mathrm{P}(\mathrm{VDF}-\mathrm{TrFE})$ nanocomposite membrane in the solar photoreactor.

The values obtained for all the wastewater measured parameters, before and after the treatment, are displayed in Table 1.

Table 1 - Main parameters of the wastewater before and after the proposed treatment. 


\begin{tabular}{|c|c|c|c|c|c|}
\hline \multicolumn{2}{|c|}{ Parameter } & $\begin{array}{c}\text { Before } \\
\text { Treatment }\end{array}$ & $\begin{array}{c}\text { After } \\
\text { Treatment }\end{array}$ & $\begin{array}{c}\text { Removed } \\
(\%)\end{array}$ & $\begin{array}{c}\text { Limit } \\
\text { Values }^{1}\end{array}$ \\
\hline \multicolumn{2}{|l|}{$\mathrm{pH}$} & 4.3 & 5.3 & - & $6.5-8.5$ \\
\hline \multicolumn{2}{|c|}{ Temperature $\left(C^{\circ}\right)$} & 21.1 & 19.1 & 9.47 & 30 \\
\hline \multicolumn{2}{|c|}{ Turbidity (NTU) } & 205 & 7 & 96.58 & 10 \\
\hline \multicolumn{2}{|c|}{$\operatorname{COD}\left(\mathrm{mg} \mathrm{l}^{-1}\right)$} & 25879 & 105 & 99.59 & 120 \\
\hline \multicolumn{2}{|c|}{ Conductivity (ms cm-1$)$} & 236 & 167.4 & 29.06 & 2 \\
\hline \multicolumn{2}{|c|}{ Chloride (mg l-1) } & 1047.4 & 804.2 & 25.82 & 500 \\
\hline \multicolumn{2}{|c|}{ Suspended solids (mg $\left.\mathrm{l}^{-1}\right)$} & 73.9 & 17.9 & 75.77 & 35 \\
\hline \multicolumn{2}{|c|}{ Total hydrocarbons (mg l-1) } & 48.69 & 0.1492 & 99.69 & 10 \\
\hline \multicolumn{2}{|c|}{ TOC $\left(\mathrm{mg} \mathrm{l}^{-1}\right)$} & 872 & 19.81 & 97.72 & 20 \\
\hline \multicolumn{2}{|c|}{ Nitrate (mg l-1) } & 440 & 137 & 68.86 & 50 \\
\hline \multicolumn{2}{|c|}{ Nitrite (mg $\left.\mathbf{l}^{-1}\right)$} & 0.99 & 0 & 100 & 1 \\
\hline \multicolumn{2}{|c|}{ Phosphate (mg l-1) } & 0.17 & 0 & 100 & 2 \\
\hline \multirow{8}{*}{$\begin{array}{l}\text { Heavy metals } \\
\qquad\left(\mathrm{mg} \mathrm{l}^{-1}\right)\end{array}$} & Cd & 9.29 & 3.49 & 62.43 & 0.2 \\
\hline & $\mathrm{Cr}$ & 1.58 & 0.75 & 52.53 & 0.1 \\
\hline & $\mathrm{Cu}$ & $<0.1$ & $<0.1$ & 100 & 3 \\
\hline & $\mathrm{Fe}$ & 817.8 & 423.4 & 48.22 & 3 \\
\hline & Mn & 552 & 385.4 & 30.25 & 1 \\
\hline & $\mathrm{Ni}$ & $<0.1$ & $<0.1$ & 100 & 0.5 \\
\hline & $\mathrm{Pb}$ & 40.55 & 28.38 & 30.01 & 0.5 \\
\hline & $\mathrm{Zn}$ & 492.9 & 290.5 & 41.06 & 3 \\
\hline
\end{tabular}

${ }^{1}$ Executive Decree No. 06-141 of 20 Rabie El Aouel 1427 corresponding to April 19, 2006, defining the limit values for discharges of industrial liquid effluents of Algeria

The results obtained after 7 hours of sunlight irradiation of the $\mathrm{TiO}_{2} / \mathrm{PVDF}-\mathrm{TrFE}$ membrane and the oily wastewater in the photoreactor show that:

- There was a reduction in all the assessed parameters, and all the final values are below the legal limits established in Algeria;

- The suspended solids concentration was removed, and only $24 \%$ remained in the solution after the treatment;

- The total hydrocarbons were removed entirely (>99\%), which indicates the efficient removal of the oil in the wastewater;

- A relevant improvement in the $\mathrm{pH}$ values of treated waters with a tendency towards a slightly acidic medium;

- The $\mathrm{TiO}_{2} / \mathrm{P}(\mathrm{VDF}-\mathrm{TrFE})$ nanocomposite was effective in the degradation of oily wastewater, as a significant decrease in the total organic carbon, COD, turbidity and heavy metal content was obtained after the treatment.

The oil degradation by the $\mathrm{TiO}_{2} / \mathrm{P}(\mathrm{VDF}-\mathrm{TrFE})$ nanocomposite can be explained based on the generation of active oxidants on $\mathrm{TiO}_{2}$ nanoparticles surface upon sunlight irradiation. These reactive species oxidised most of the organic compounds found in the oily water. The removal 
of $99 \%$ of the HC, confirmes the efficient degradation of the contaminants. This is also confirmed by the disappearance of peaks in the chromatogram obtained by gas chromatographic analysis (GC-FID), Figure 3.
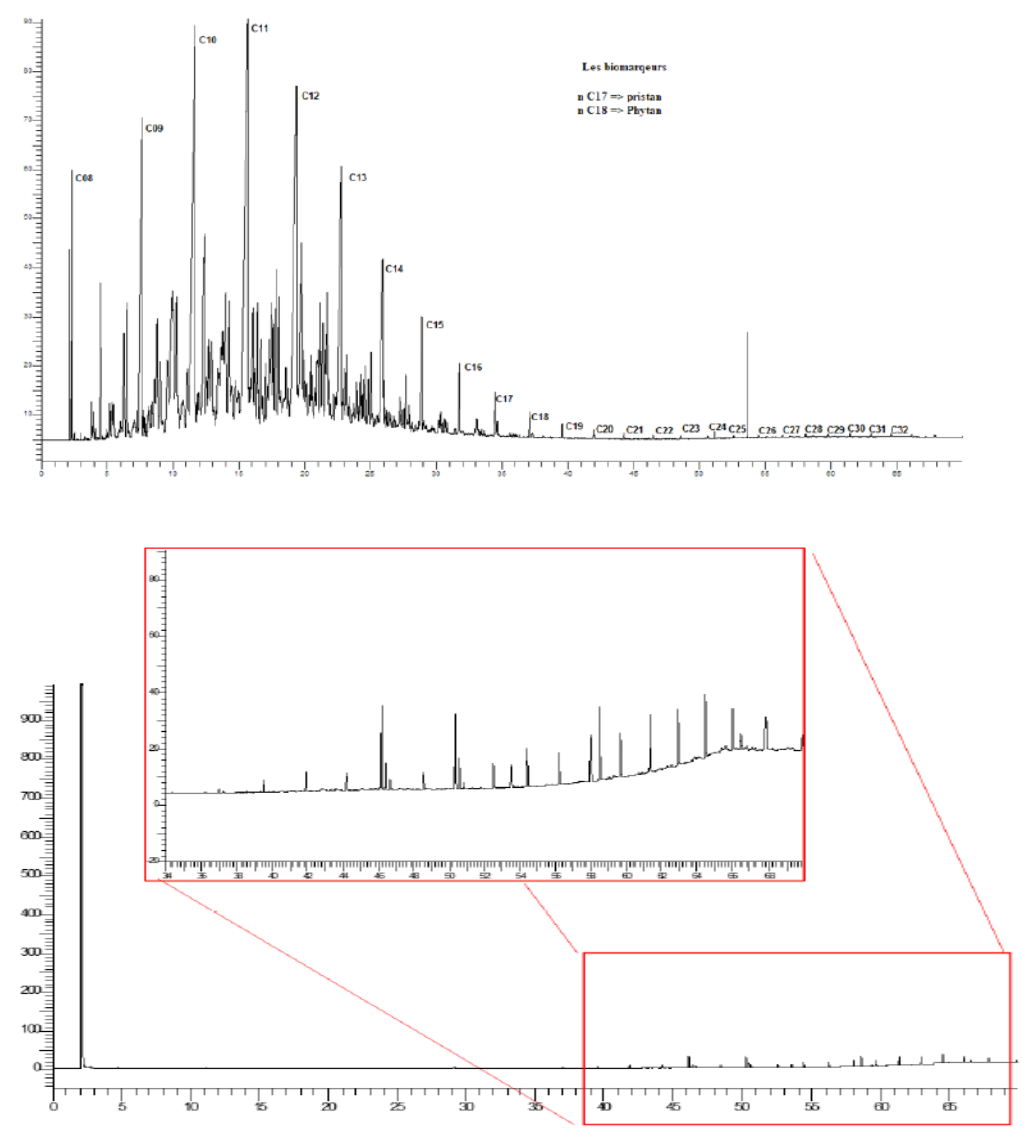

Figure 3 - Chromatogram corresponding to the sample before (a) and after (b) the photocatalytic degradation in 7 hours of sunlight irradiation.

The majority of the organic compounds present in the sample before treatment (Figure 3a) belong to the alkanes (C10-C30), which is in good agreement with similar works [6] [20]. After the photocatalytic treatment the presence of all these compound was reduced, Figure $5 \mathrm{~b}$. The long chain hydrocarbons were degraded by photocatalytic activity into smaller organic compounds.

The results show that using the $\mathrm{TiO} 2 / \mathrm{P}(\mathrm{VDF}-\mathrm{TrFE})$ nanocomposite membrane; it is possible to treat oily industrial wastewater, such as the one from the central region of Hassi Rmel Algeria, using the sun as a source of renewable energy.

\section{Materials and Methods}

\subsection{Materials}


Poly(vinylidene fluoride-trifluoroethylene), (P(VDF-TrFE)) 70:30 was purchased to Solvay (Belgium). Evonik Industries kindly provided titanium dioxide (TiO2) nanoparticles (P25). The oily water was withdrawn from the central industrial region of Hassi R'mel Algeria.

\subsection{Membrane preparation and characterisation}

The solvent casting technique was used to produce the poly(vinylidene difluoride)-cotrifluoroethylene $(\mathrm{P}(\mathrm{VDF}-\mathrm{TrFE}))$ membranes with immobilised $\mathrm{TiO}_{2}$ nanoparticles $[18,19]$. Briefly, $0.86 \mathrm{~g}$ ( $8 \mathrm{wt} . \%$ ) of $\mathrm{TiO}_{2}$ nanoparticles were added to $90 \mathrm{ml}$ of $\mathrm{N}, \mathrm{N}$-dimethylformamide (DMF, Merck) and placed in an ultrasound bath for four hours to obtain a homogeneously dispersed solution. The amount of $\mathrm{TiO}_{2}$ nanoparticles used was previously chosen [19] to prevent detachment from the polymer matrix and to preserve its mechanical properties. Then, $10 \mathrm{~g}$ of P(VDF-TrFE) was added to the solution, achieving a concentration of $10 \mathrm{wt} . \%$ polymer to solvent, and the solution was magnetically stirred until complete polymer dissolution. Ultimately, the solution was placed in glass support, and the solvent evaporated at ambient temperature. When the solvent completely evaporated, a membrane with the same dimensions of the photoreactor surface $(38 \mathrm{~cm}$ length $\times 12 \mathrm{~cm}$ wide $\times \approx 600 \mu \mathrm{m}$ thick) was precisely cut out.

The crystal structure of the $\mathrm{TiO}_{2}$ nanoparticles was evaluated by X-ray diffraction (XRD). Fourier transformed infrared spectroscopy (FTIR), and scanning electron microscopy (SEM) were performed to respectively access the chemical structure and morphology/microstructure of the membrane. The wettability of the samples was obtained through contact angle measurements.

\subsection{Oily wastewater sampling and parameters}

The Hassi R'mel (HR) area is located in southern Algeria, $525 \mathrm{~km}$ south of Algiers, and $120 \mathrm{~km}$ south of Laghouat wilaya, about $70 \mathrm{~km}$ west of Beriane and $120 \mathrm{~km}$ south of Algiers, northwest of Ghardaia. The HR field is centred at $32^{\circ} 55^{\prime} 41^{\circ}$ North and $3^{\circ} 16^{\prime} 16^{\circ}$ East at an average elevation of approximately 750 meters above sea level. The geometric shape, the nature of the effluent and the homogeneity of the reservoir at the HR field led to the selection of three production areas (North, Center, and South) and two compressor stations.

The withdraw of samples was accomplished on 15 March 2017, at the inlet of the deoiling unit at the CPI S-102 (Corrugated Plate Interceptor), which is a separating basin furnished with a set of parallel plates that facilitate the sedimentation of the non-decanted solid particles. The samples were transported from Hassi R'mel to the Research and Development Center in coolers and stored in a refrigerator at $4{ }^{\circ} \mathrm{C}$.

Several parameters were measured before and after the treatment. The temperature $(\mathrm{T})$ was measured using a thermometer ASTM 5C and the electrical conductivity (EC) was measured by a conductivity meter HACH HQ 40d. The suspended solids (SS) were determined by filtering $100 \mathrm{ml}$ of water to be analysed through a $47 \mu \mathrm{m}$ pore filter; afterwards, the filter was dried at $105^{\circ} \mathrm{C}$ in a hoven for 2 hours. The amount of suspended solids was estimated by the difference in the weight of the filter before and after drying. The $\mathrm{pH}$ measurements were carried out using a $\mathrm{pH}$-meter type (Inolab PH7310). Chemical oxygen demand (COD) is based on the oxidation of organic materials by an excess of potassium dichromate ( $\mathrm{K} 2 \mathrm{Cr} 2 \mathrm{O} 7)$, in acidic medium and boiling, in the presence of silver sulfate and mercury sulfate. COD is measured by a spectrophotometer of the type (DR 1900 LANGE HACH). 
The quantification of the total hydrocarbons (HC) in water followed an analytical protocol implemented according to the water standard methods (solvent extraction, purification on florisil cartridge, and finally gas chromatography analysis coupled to a flame ionisation detector - Clarus 580 CPG/FID).

The Total Organic Carbon (TOC) is one of the quality control criteria for water used to measure biological impurities (organic matter) present in water. For TOC analysis, the infrared analyser performs the dosage in two stages: Determination of total carbon (TC) and determination of inorganic carbon (IC). Total organic carbon is the difference between total carbon (TC) and inorganic carbon (IC), TOC was analysed by TOC Analyzer Formacs HT.

The determination of phosphate, nitrate, and nitrite was carried out using a DR1900 colourimetric spectrophotometer at a wavelength of $860 \mathrm{~nm}$ using the different LCK reagents.

Heavy metals presence and quantification were performed using a Spectrophotometer ICP 8000 DV.

\subsection{Photocatalytic activity measurments}

The photocatalytic degradation of oily wastewater was performed in a solar photoreactor located in the north of Algeria (latitude $36.39^{\circ}$; longitude $2.42^{\circ}$ at sea level), using natural sunlight radiation. A Pyranometer CMP 11 (Kipp \& Zonen) with a spectral range between 285 and $2800 \mathrm{~nm}$ was used to measure the solar UV intensity. The reactor was developed at the Solar Equipment Development Unit (UDES) in Algeria. The capacity of the photoreactor is 11 ( $38 \mathrm{~cm}$ length $\times 12 \mathrm{~cm}$ wide $\times 8.5 \mathrm{~cm}$ high). The photoreactor tank was fabricated from Pyrex glass, where the produced TiO2/PVDF-TrFE nanocomposite membrane was placed at the bottom (Figure 4).
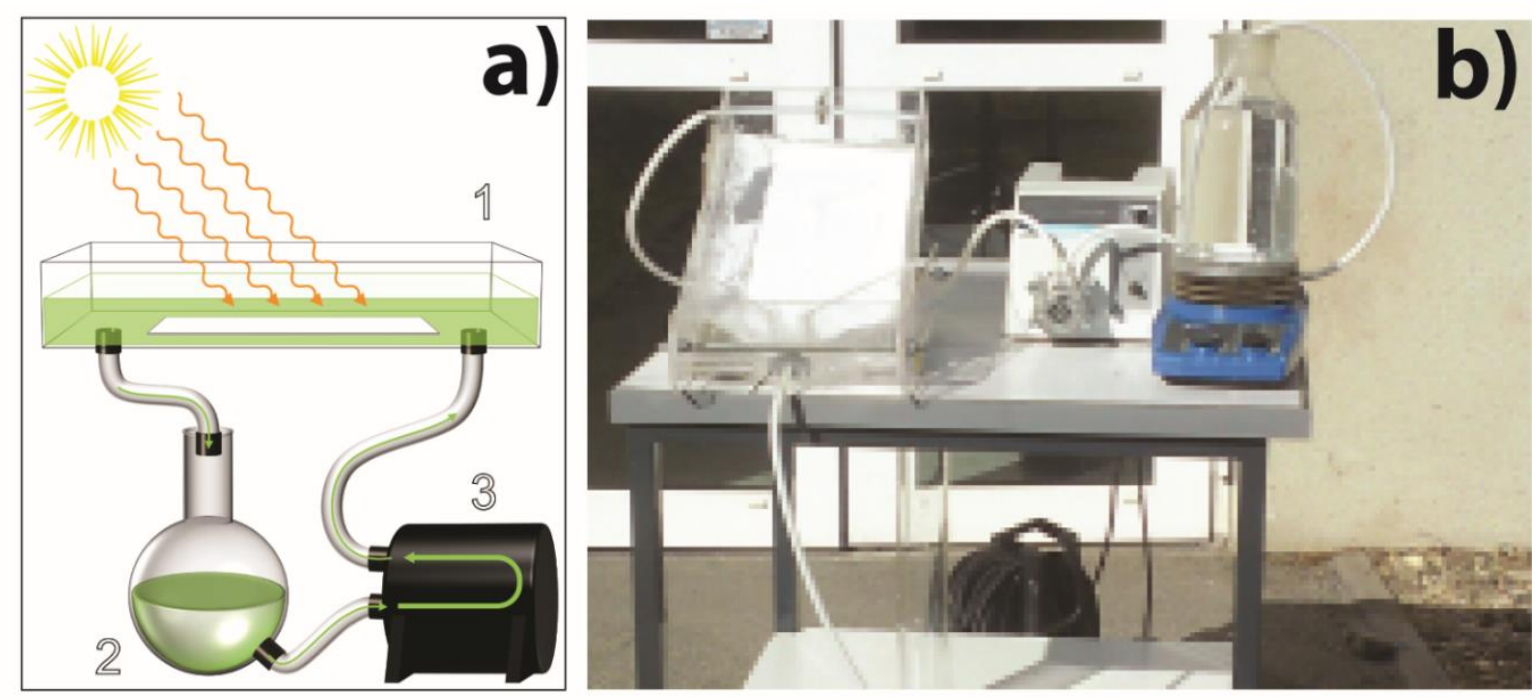

Figure 4 - Representation of the solar photoreactor:1- photoreactor tank with the photocatalytic membrane at the bottom; 2 - oily wastewater; 3- peristaltic pump a); picture of the photoreactor setup b).

The flow rate used to recirculate the oily water was $28 \mathrm{ml} \mathrm{s}-1$; in this way, it was possible to cover the nanocomposite membrane with this solution completely. The photoreactor was wholly covered with glass to avoid evaporation during the photocatalytic experiments. For the photocatalytic assays, one liter of oily wastewater solution was added to the photoreactor tank containing the membrane and exposed to solar illumination for 7 hours. 


\section{Conclusions}

In short, this work reports the preparation of a $\mathrm{TiO}_{2} / \mathrm{P}(\mathrm{VDF}-\mathrm{TrFE})$ nanocomposite by solvent casting and its photocatalytic application in the remediation of oily wastewater. The characterization has shown a suitable porous structure and a homogeneous distribution of the $\mathrm{TiO}_{2}$ nanoparticles over the interconnected pores. Also, the photocatalytic efficiency on the degradation of oil in oily wastewater was tested under sunlight irradiation, showing significant efficiency. Colorless water was obtained after the oily wastewater exposure to visible light for 7 hours. Moreover, other relevant parameters were improved, namely the remarkable reduction of nitrate, phosphate, suspended solids, total hydrocarbons, and TOC degradation. In the scope of water remediation, the removal of several heavy metals should also be highlighted. In conclusion, the $\mathrm{TiO}_{2} / \mathrm{P}(\mathrm{VDF}-\mathrm{TrFE})$ nanocomposite membrane is a suitable solution to oily wastewater remediation.

Author Contributions: Methodology D.Z. and L.D.; investigation D.Z., L.D., H.S., and P.M.M.; analysis L.D. and P.M.M.; writing - original draft preparation D.Z.,H.S., P.M.M; writing - review and editing S.L.M; project administration SLM. ${ }^{+D}$. Zioui and H. Salazar contributed equally to this work.

Funding: This research received no external funding.

Acknowledgments: This work was supported by the Solar Equipment Development Unit (UDES) Algeria. This work was also supported by the Portuguese Foundation for Science and Technology (FCT) in the framework of the strategic project UID/FIS/04650/2013 by FEDER funds through the COMPETE 2020-Programa Operacional Competitividade e Internacionalização (POCI) with the reference project POCI-01-0145-FEDER-006941, and project PTDC/CTM-ENE/5387/2014. P.M. Martins and H. Salazar thanks the FCT for the grants SFRH/ BD/ 98616/2013 and SFRH/BD/122373/2016. The authors acknowledge funding from the Basque Government Industry Department under the ELKARTEK and HAZITEK programs and the Spanish Ministry of Economy and Competitiveness (MINECO) through the project MAT2016-76039-C4-3-R(AEI/FEDER, UE) (including the FEDER financial support).

Conflicts of Interest: The authors declare no conflicts of interest.

\section{References}

1. Rusli, U.N.; Alias, N.H.; Shahruddin, M.Z.; Othman, N.H. Photocatalytic Degradation of Oil using Polyvinylidene Fluoride/Titanium Dioxide Composite Membrane for Oily Wastewater Treatment. MATEC Web Conf. 2016, 69, 05003.

2. Sun, S.; Xiao, Q.R.; Zhou, X.; Wei, Y.Y.; Shi, L.; Jiang, Y. A bio-based environment-friendly membrane with facile preparation process for oil-water separation. Colloids and Surfaces $A$ : Physicochemical and Engineering Aspects 2018, 559, 18-22, doi:10.1016/j.colsurfa.2018.09.038. 
3. Stack, L.J.; Carney, P.A.; Malone, H.B.; Wessels, T.K. Factors influencing the ultrasonic separation of oil-in-water emulsions. Ultrasonics Sonochemistry 2005, 12, 153-160, doi:10.1016/j.ultsonch.2003.10.008.

4. Soares, S.F.; Rodrigues, M.I.; Trindade, T.; Daniel-da-Silva, A.L. Chitosan-silica hybrid nanosorbents for oil removal from water. Colloids and Surfaces A: Physicochemical and Engineering Aspects 2017, 532, 305-313, doi:10.1016/j.colsurfa.2017.04.076.

5. Cañizares, P.; Martínez, F.; Jiménez, C.; Sáez, C.; Rodrigo, M.A. Coagulation and electrocoagulation of oil-in-water emulsions. Journal of Hazardous Materials 2008, 151, 4451, doi:10.1016/j.jhazmat.2007.05.043.

6. Ong, C.S.; Lau, W.J.; Goh, P.S.; Ng, B.C.; Ismail, A.F. Investigation of submerged membrane photocatalytic reactor (sMPR) operating parameters during oily wastewater treatment process. Desalination 2014, 353, 48-56, doi:https://doi.org/10.1016/i.desal.2014.09.008.

7. Salazar, H.; Nunes-Pereira, J.; Correia, D.M.; Cardoso, V.F.; Gonçalves, R.; Martins, P.M.; Ferdov, S.; Martins, M.D.; Botelho, G.; Lanceros-Méndez, S. Poly(vinylidene fluoridehexafluoropropylene)/bayerite composite membranes for efficient arsenic removal from water. Materials Chemistry and Physics 2016, 183, 430-438, doi:10.1016/j.matchemphys.2016.08.049.

8. Zhang, X.; Wang, Y.; Liu, Y.; Xu, J.; Han, Y.; Xu, X. Preparation, performances of PVDF/ZnO hybrid membranes and their applications in the removal of copper ions. Applied Surface Science 2014, 316, 333-340, doi:https://doi.org/10.1016/j.apsusc.2014.08.004.

9. Song, H.; Shao, J.; Wang, J.; Zhong, X. The removal of natural organic matter with LiCl-TiO2doped PVDF membranes by integration of ultrafiltration with photocatalysis. Desalination 2014, 344, 412-421, doi:https://doi.org/10.1016/j.desal.2014.04.012.

10. Zhang, M.; Zhang, L.; Cheng, L.-H.; Xu, K.; Xu, Q.-P.; Chen, H.-L.; Lai, J.-Y.; Tung, K.-L. Extracorporeal endotoxin removal by novel I-serine grafted PVDF membrane modules. Journal of Membrane Science 2012, 405-406, 104-112, doi:https://doi.org/10.1016/j.memsci.2012.02.057.

11. Ramaiah, K.P.; Satyasri, D.; Sridhar, S.; Krishnaiah, A. Removal of hazardous chlorinated VOCs from aqueous solutions using novel ZSM-5 loaded PDMS/PVDF composite membrane consisting of three hydrophobic layers. Journal of Hazardous Materials 2013, 261, 362-371, doi:https://doi.org/10.1016/j.jhazmat.2013.07.048.

12. Zuo, X.; Shi, W.; Tian, Z.; Yu, S.; Wang, S.; He, J. Desalination of water with a high degree of mineralization using SiO2/PVDF membranes. Desalination 2013, 311, 150-155, doi:https://doi.org/10.1016/j.desal.2012.11.034.

13. Aoudjit, L.; Martins, P.M.; Madjene, F.; Petrovykh, D.Y.; Lanceros-Mendez, S. Photocatalytic reusable membranes for the effective degradation of tartrazine with a solar photoreactor. Journal of Hazardous Materials 2018, 344, 408-416, doi:https://doi.org/10.1016/i.jhazmat.2017.10.053.

14. Martins, P.M.; Gomez, V.; Lopes, A.C.; Tavares, C.J.; Botelho, G.; Irusta, S.; Lanceros-Mendez, S. Improving photocatalytic performance and recyclability by development of Er-doped and Er/Pr-codoped TiO2/Poly(vinylidene difluoride)-trifluoroethylene composite membranes. Journal of Physical Chemistry C 2014, 118, 27944-27953, doi:10.1021/jp509294v.

15. Martins, P.M.; Miranda, R.; Marques, J.; Tavares, C.J.; Botelho, G.; Lanceros-Mendez, S. Comparative efficiency of $\mathrm{TiO}<\mathrm{inf}>2</ \mathrm{inf}>$ nanoparticles in suspension vs. immobilization 
into P(VDF-TrFE) porous membranes. RSC Advances 2016, 6, 12708-12716, doi:10.1039/c5ra25385c.

16. Zhang, W.; Ding, L.; Luo, J.; Jaffrin, M.Y.; Tang, B. Membrane fouling in photocatalytic membrane reactors (PMRs) for water and wastewater treatment: A critical review. Chemical Engineering Journal 2016, 302, 446-458, doi:10.1016/j.cej.2016.05.071.

17. Jin, Z.; Duan, W.; Liu, B.; Chen, X.; Yang, F.; Guo, J. Fabrication of efficient visible light activated $\mathrm{Cu}-\mathrm{P} 25$-graphene ternary composite for photocatalytic degradation of methyl blue. Applied Surface Science 2015, 356, 707-718, doi:http://dx.doi.org/10.1016/i.apsusc.2015.08.122.

18. Ribeiro, C.; Costa, C.M.; Correia, D.M.; Nunes-Pereira, J.; Oliveira, J.; Martins, P.; Gonçalves, R.; Cardoso, V.F.; Lanceros-Méndez, S. Electroactive poly(vinylidene fluoride)-based structures for advanced applications. Nature Protocols 2018, 13, 681, doi:10.1038/nprot.2017.157

\section{https://www.nature.com/articles/nprot.2017.157\#supplementary-information.}

19. Martins, P.M.; Miranda, R.; Marques, J.; Tavares, C.J.; Botelho, G.; Lanceros-Mendez, S. Comparative efficiency of TiO2 nanoparticles in suspension vs. immobilization into P(VDFTrFE) porous membranes. RSC Advances 2016, 6, 12708-12716, doi:10.1039/C5RA25385C.

20. Li, G.; An, T.; Chen, J.; Sheng, G.; Fu, J.; Chen, F.; Zhang, S.; Zhao, H. Photoelectrocatalytic decontamination of oilfield produced wastewater containing refractory organic pollutants in the presence of high concentration of chloride ions. Journal of Hazardous Materials 2006, 138, 392-400, doi:https://doi.org/10.1016/j.jhazmat.2006.05.083. 